\title{
Identification of advection-diffusion equation from a limited number of spatial locations
}

\author{
J. Schorsch ${ }^{1,2}$, M. Gilson ${ }^{1,2}$, H. Garnier ${ }^{1,2}$ \\ ${ }^{1}$ University of Lorraine, CRAN, UMR 7039, 2 rue Jean Lamour, \\ F-54519 Vandoeuvre-les-Nancy, France \\ ${ }^{2}$ CNRS, CRAN, UMR 7039, France \\ julien.schorsch, marion.gilson, hugues.garnier@univ-lorraine.fr
}

\begin{abstract}
This paper presents an instrumental variable method for identifying advectiondiffusion equation models in presence of output measurement noise. This partial differential equation is commonly used to describe the transport and dispersion of a solute in a river channel. The realistic measurement situation where spatial data are limited and non-uniformly distributed is considered. A refined instrumental variable method involving adaptive data filtering is proposed to handle the non-uniformly spatial data distribution. The performance of the proposed approach are illustrated by a numerical example.
\end{abstract}

\section{INTRODUCTION}

Heat conduction, state-of-health in advances batteries or population dynamics are phenomena described by partial differential equation (PDE). PDE are differential equations that contain multivariable parameterized input/output functions and their derivatives. These functions are continuous functions which can depend on a spatial location point and on a time instant.

This paper focuses on a special case of PDE: the advectiondiffusion equation (ADE). More precisely the coefficient estimation problem of the ADE is considered here. This equation is often used in the water resource quality analysis to describe the transport and dispersion of a solute (pesticide, pollutant, ...) in a river channel (see e.g. Martinez and Wise [2003]).

Various different techniques have been proposed to identify the parameters in ADE models. Tych and Young [2012] have developed an approach to emulate the dynamic behaviour of ADE using a continuous-time transfer function model for each space-point. In Nilssen et al. [2009], the augmented Lagrangian method is utilized to estimate the diffusion coefficient.

In practical situations, such as those encountered in environmental science, the measured data are not continuous in time and in space. Sampled data are indeed measured from sensors which are usually not uniformly placed leading to non-uniform data distribution in space. Moreover, due to the sensor breakdown or data-storage errors, data can also be missing in time. The signals are then measured at varying sampling rates.

Several techniques for PDE model identification in case of missing data have already been proposed. In Hidayat et al. [2012] an iterative scheme is used to impute the missing data based on a multivariable prediction model identified from measurements of neighboring sensors. An identification of coupled map lattice models of complex spatio-temporal patterns using a limited number of spatial locations is proposed in Coca and Billings [2001]. The problem of non-uniform sampling data can also be considered by a discrete-time equivalent model identification using a linear-parameter-varying method with parameters depending on the sampling (see e.g. Ali et al. [2011]).

This paper investigates a method for identifying continuous ADE model from a limited number of irregularly spatial locations. The proposed method presented here is an extension of the Simplified Refined Instrumental Variable for PDE model identification (SRIVPDE) (see Schorsch et al. [2012]). One advantage of this approach to continuous modeling is to be able to handle the case of irregularly spatial data. This method is based on the instrumental variable (IV) technique involving an adaptive data prefiltering. Moreover, this paper demonstrates the interest of this method to emulate the DPS.

This paper is organized as follows. Section 2 formulates the identification problem. In Section 3 the proposed method is developed. The digital implementation of the continuous-time filtering operations involved in the proposed ADE model estimation scheme is described in Section 4. Numerical simulation examples are used in Section 5 in order to illustrate the effectiveness of the proposed method. Finally, Section 6 presents the main conclusions.

\section{PROBLEM FORMULATION}

Let $\Omega$ an open set included in $\mathbb{R}^{\mathrm{M}}$ with $\mathrm{M}$ an integer and $\Gamma$ the boundary of $\Omega$, and consider a space-time continuous system defined by the ADE described as follows:

$$
\left\{\begin{array}{l}
\left.\left.\frac{\partial \stackrel{\circ}{y}(x, t)}{\partial t}=a_{20}^{\mathrm{o}} \frac{\partial^{2} \stackrel{\circ}{y}(x, t)}{\partial x^{2}}-a_{10}^{\mathrm{o}} \frac{\partial \stackrel{\circ}{y}(x, t)}{\partial x} \text { on } \Omega \times\right] 0, T\right] \\
\stackrel{\circ}{y}(x, t)=\stackrel{\circ}{\leftarrow}_{\Gamma}(x, t) \text { on } \Gamma \times[0, T] \\
\stackrel{\circ}{y}(x, 0)=\stackrel{\circ}{y}_{0}(x) \text { on } \Omega
\end{array}\right.
$$


where $\stackrel{y}{y}(x, t)$ is a space-time continuous function defined as follows

$$
\grave{y}(x, t): \bar{\Omega} \times[0, T] \rightarrow \mathbb{R}
$$

where $\bar{\Omega}=\Omega \cup \Gamma . \stackrel{\circ}{y}_{0}(x)$ and $\stackrel{\circ}{u}_{\Gamma}(x, t)$ are given functions which represent the initial condition and the boundary conditions respectively. $x \in \bar{\Omega}$ is the space point and $t \in[0, T]$ is the time variable.

In (1), $a_{20}^{\mathrm{o}}$ represents the dispersion coefficient while $a_{10}^{\mathrm{o}}$ denotes the volumetric flow.

The function $\stackrel{\circ}{y}(x, t)$ is assumed to be sampled in time and space. It is therefore necessary to mesh the domain where the ADE is defined. For simplicity and without loss of generality, a simple one-dimensional problem is considered here $(M=1)$. To represent the most practical case where the data are non-uniformly measured, it is first assumed a non-uniform meshing of $\bar{\Omega}=\left[0, x_{\mathrm{L}}\right]$ where $x_{\mathrm{L}}$ is the channel length. The interval $\left[0, x_{L}\right]$ is then divided into $L$ intervals of different lengths $h_{\ell}=x_{\ell}-x_{\ell-1}$ (see Fig. 1) with $h_{0}=0$. $h_{\ell}$ may be viewed as the distance between two sensors or two observation points along the channel.

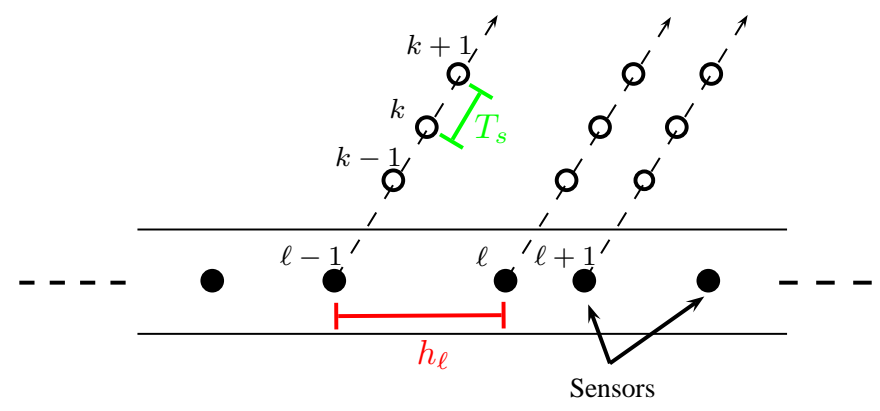

Fig. 1. Sensors positions

The second hypothesis assumes a uniform subdivision of time interval $[0, T]$ into $N$ intervals of length $T_{s}>0$. The time sampling period $T_{s}$ is supposed here to be constant:

$$
t_{k}=k T_{s} \text { for } 0 \leq k \leq N \text {, and } T_{s}=T / N
$$

As a result, the samples defined as

$$
\left(x_{\ell}, t_{k}\right)=\left(\sum_{j=0}^{j=\ell} h_{j}, k T_{s}\right)
$$

for $\ell \in\{0, \cdots, L\}, 0 \leq k \leq N$, represent the points where the functions are sampled.

The sampled signal is then denoted as $y\left(x_{\ell}, t_{k}\right)$ and the output observation equation then takes the form,

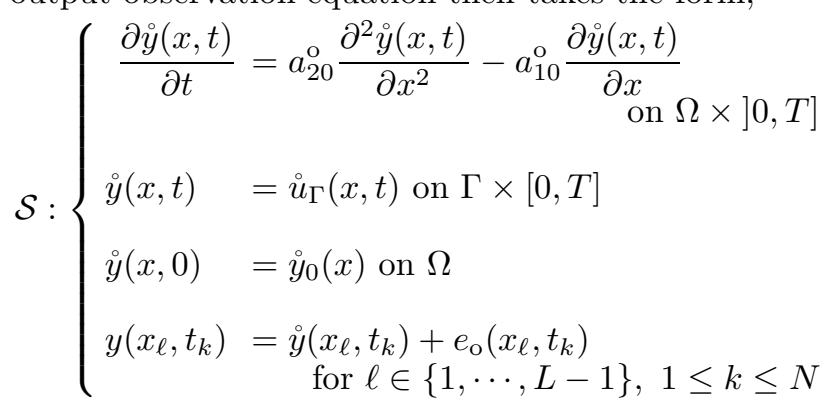

where $\stackrel{y}{ }\left(x_{\ell}, t_{k}\right)$ is the sampled value of the unobserved, noise-free output $\stackrel{\circ}{y}(x, t)$. The additive noise $e_{o}\left(x_{\ell}, t_{k}\right)$ is assumed to be a zero mean, random spatial array, with no temporal or spatial correlation.

The system is represented as follows

$$
\mathcal{G}:\left\{\begin{array}{l}
G\left(p_{x}, p_{t}, \theta\right)=\frac{1}{A\left(p_{x}, p_{t}, \theta\right)} \\
\text { with } A\left(p_{x}, p_{t}, \theta\right)=p_{t}-a_{20} p_{x}^{2}+a_{10} p_{x}
\end{array}\right.
$$

where $p_{x}$ and $p_{t}$ denote the differential operator

$$
p_{x}=\frac{\partial}{\partial x}, \quad p_{t}=\frac{\partial}{\partial t}
$$

and

$$
\theta=\left[\begin{array}{ll}
a_{20} & a_{10}
\end{array}\right]^{T}
$$

is the parameter vector that characterizes the model.

Finally, the identification model can be written in the form

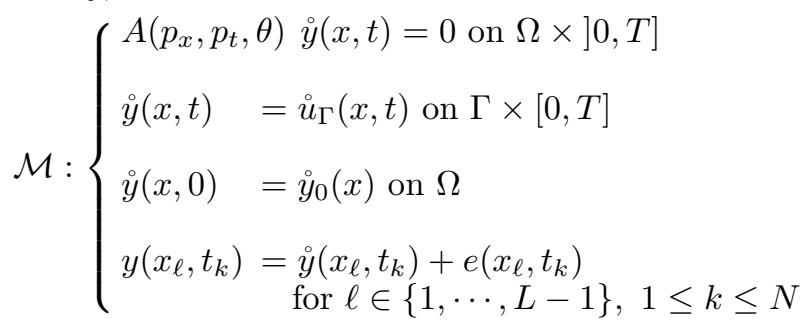

The objective is then to estimate the parameters (7) of the model (8) based on the non-uniformly sampled data $Z^{(L-2) \times(N-1)}=\left\{y\left(x_{\ell}, t_{k}\right)\right\}_{\ell=1, k=1}^{L-1, N}$ and the initial and boundary conditions.

\section{LS AND REFINED IV ESTIMATORS}

To solve the identification problem of systems described by PDE, several methods are available and two main approaches can be distinguished: the indirect and the direct approaches. The indirect approach consists in estimating the discrete formulation of the PDE (see Section 4). In that case, the parameters to be estimated are those of the discretized PDE model, and therefore they depend on spatial and time sampling. In another words, the resulting model is only valid for the experimental data used for the identification. As a result, this indirect approach cannot be directly applied neither for the non-uniformly data case, nor for the missing data case. The direct approach consists in directly identifying the continuous space-time PDE model coefficients from the available sampled data. The main advantage of this method is to provide a model which is not dependent on the space and time sampling intervals. Unlike the discretized PDE model where only sampled input and output data appear, the direct method is based on the continuous PDE written in terms of partial derivatives of these signals which are usually not available from measurement. This problem is, however, well-known and various filtering methods have been devised to handle the partial derivatives.

\subsection{The LS estimator}

Let us first consider the identification problem in the noisefree case. The ADE in (8) takes the form

$$
p_{t} \stackrel{\circ}{y}(x, t)=a_{20} p_{x}^{2} \stackrel{\circ}{y}(x, t)-a_{10} p_{x} \stackrel{\circ}{y}(x, t)
$$


Let $F\left(p_{x}, p_{t}\right)$ be a filter applied to (9),

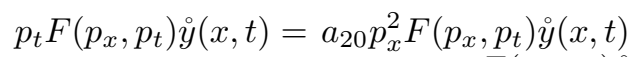

$$
\begin{aligned}
& -a_{10} p_{x} F\left(p_{x}, p_{t}\right) \grave{y}(x, t)
\end{aligned}
$$

Let $F^{\left(i_{x}, i_{t}\right)}\left(p_{x}, p_{t}\right)$ for $i_{x}=0,1,2$ and $i_{t}=0,1$ be a set of filters defined as

$$
F^{\left(i_{x}, i_{t}\right)}\left(p_{x}, p_{t}\right)=p_{x}^{i_{x}} p_{t}^{i_{t}} F\left(p_{x}, p_{t}\right)
$$

When considering the filter initial and boundary conditions both null, and by using the filters defined in (11), (10) can be rewritten as

$$
\dot{y}_{\mathrm{f}}^{(0,1)}(x, t)=a_{20} \stackrel{\circ}{\mathrm{f}}_{\mathrm{f}}^{(2,0)}(x, t)-a_{10} \stackrel{\circ}{\mathrm{f}}_{\mathrm{f}}^{(1,0)}(x, t)
$$

where

$$
{\stackrel{\circ}{y_{\mathrm{f}}}}^{\left(i_{x}, i_{t}\right)}(x, t)=F^{\left(i_{x}, i_{t}\right)}\left(p_{x}, p_{t}\right) \grave{y}(x, t)
$$

At time-instant $t=t_{k}$ and for a space point $x=x_{\ell}$, considering now the situation where an additive noise is corrupting the output measurement, equation (12) can be rewritten in linear regression form as

$$
y_{\mathrm{f}}^{(0,1)}\left(x_{\ell}, t_{k}\right)=\varphi_{\mathrm{f}}^{T}\left(x_{\ell}, t_{k}\right) \theta+\epsilon\left(x_{\ell}, t_{k}\right)
$$

with

$$
\varphi_{\mathrm{f}}^{T}\left(x_{\ell}, t_{k}\right)=\left[y_{\mathrm{f}}^{(2,0)}\left(x_{\ell}, t_{k}\right)-y_{\mathrm{f}}^{(1,0)}\left(x_{\ell}, t_{k}\right)\right]
$$

The LS estimator for ADE models (denoted as LSADE in the sequel) can be computed from the following estimates

$$
\begin{aligned}
\hat{\theta}_{\mathrm{LSADE}}=\left[\sum_{\ell=0}^{L} \sum_{k=0}^{N} \varphi_{\mathrm{f}}\left(x_{\ell}, t_{k}\right) \varphi_{\mathrm{f}}^{T}\left(x_{\ell}, t_{k}\right)\right]^{-1} . & {\left[\sum_{\ell=0}^{L} \sum_{k=0}^{N} \varphi_{\mathrm{f}}\left(x_{\ell}, t_{k}\right) y_{\mathrm{f}}^{(0,1)}\left(x_{\ell}, t_{k}\right)\right] }
\end{aligned}
$$

provided that the matrix inverse exists.

However, it is well-known that such LSADE parameter estimates are asymptotically biased and statistically inconsistent in the general practical situation where the spatio-temporal output measurements are contaminated by measurement noise even if, as in this case, the noise is assumed to be zero mean and completely random in nature. A traditional method to remove the asymptotic bias from the LS estimates is to use instrumental variable (IV) techniques [see Söderström and Stoica [1983] for discrete-time models and Young [1984, 2011] for discrete and continuous-time models].

\section{Comments:}

One traditional filter described in Schorsch et al. [2012] is the state variable filtering (SVF). The same SVF filter is used here, defined as

$$
F\left(p_{x}, p_{t}\right)=\frac{1}{\left(p_{t}+\lambda_{x}\right)^{2}\left(p_{t}+\lambda_{t}\right)}
$$

where $\lambda_{x}$ and $\lambda_{t}$ are the breakpoint frequencies in space and time respectively.

\subsection{The IV estimator}

\subsubsection{Principle}

The main idea behind the IV method is to modify the normal equations (16) so that they yield consistent estimates for arbitrary additive noise. This involves the introduction of an IV vector $\zeta\left(x_{\ell}, t_{k}\right)$ whose elements are correlated with the filtered noise-free regression vector $\dot{\varphi}\left(x_{\ell}, t_{k}\right)$, defined as

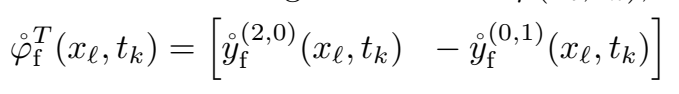

but uncorrelated with the additive noise. The basic IV parameter estimates are then given by the following IV normal equations:

$$
\begin{array}{r}
\hat{\theta}_{\mathrm{IV}}=\left[\sum_{\ell=0}^{L} \sum_{k=0}^{N} \zeta_{\mathrm{f}}\left(x_{\ell}, t_{k}\right) \varphi_{\mathrm{f}}^{T}\left(x_{\ell}, t_{k}\right)\right]^{-1} \cdot \\
{\left[\sum_{\ell=0}^{L} \sum_{k=0}^{N} \zeta_{\mathrm{f}}\left(x_{\ell}, t_{k}\right) y_{\mathrm{f}}^{(0,1)}\left(x_{\ell}, t_{k}\right)\right]}
\end{array}
$$

provided that the matrix inverse exists.

The IV method provides a consistent estimate of $\theta$ if

$$
\left\{\begin{array}{l}
E\left[\zeta_{\mathrm{f}}\left(x_{\ell}, t_{k}\right) \varphi_{\mathrm{f}}^{\mathrm{T}}\left(x_{\ell}, t_{k}\right)\right] \quad \text { is not singular } \\
E\left[\zeta_{\mathrm{f}}\left(x_{\ell}, t_{k}\right) v_{o}\left(x_{\ell}, t_{k}\right)\right]=0
\end{array}\right.
$$

where $E($.$) denotes the mathematical expectation, and$ $v_{o}\left(x_{\ell}, t_{k}\right)$ is defined as follow

$$
v_{o}\left(x_{\ell}, t_{k}\right)=F\left(p_{x}, p_{t}\right) e_{o}\left(x_{\ell}, t_{k}\right)
$$

\subsubsection{SRIVADE estimator}

We recall the main conditions for obtaining optimal (consistent and minimum variance) parameter estimate when using IV method in the case of ordinary differential equation models. The statistically optimal IV is critically dependent on the following selection of the IV vector $\zeta\left(t_{k}\right)$ and the prefilter $F(p)$ [see e.g. Söderström and Stoica, 1983, Young, 1984, Young et al., 2008, Young, 2011]:

$$
\left\{\begin{array}{l}
\zeta_{\mathrm{f}}^{o p t}\left(t_{k}\right)=F^{o p t}\left(p_{t}\right) \dot{\varphi}\left(t_{k}\right) \\
F^{o p t}\left(p_{t}\right)=\frac{1}{A_{o}\left(p_{t}\right)}
\end{array}\right.
$$

where $\dot{\varphi}\left(t_{k}\right)$, which is the single dimensional equivalent of $\grave{\varphi}\left(x_{\ell}, t_{k}\right)$ in (18), is constructed from the noise-free signals. The optimal Simplified Refined Instrumental Variable for Continuous-time systems (SRIVC) method, first developed by Young and Jakeman [1980] and later extended to the more general case of coloured noise disturbances by Young et al. [2008] is based on these theoretical considerations.

Using the same idea as in the SRIVC method for PDE model (see Schorsch et al. [2012]), a new estimator is proposed here to handle the ADE case and will be denoted as SRIVADE in the sequel. The conditions to obtain optimal estimates become:

$$
\left\{\begin{array}{l}
\zeta_{\mathrm{f}}^{o p t}\left(x_{\ell} t_{k}\right)=F^{o p t}\left(p_{x}, p_{t}\right) \dot{\varphi}\left(x_{\ell}, t_{k}\right) \\
F^{o p t}\left(p_{x}, p_{t}\right)=\frac{1}{A_{o}\left(p_{x}, p_{t}\right)}
\end{array}\right.
$$

Both the prefilter and instruments require the knowledge of the true plant model. This is a usual dilemma encountered in the problem of accuracy optimization. The 
proposed refined IV method uses an iterative procedure, in which, at each iteration, the auxiliary model is used to generate the instrumental variables and prefilter based on the parameters obtained at the previous iteration.

\section{- Step 1 Initialisation}

Generate an initial estimate of the TF model parameter vector $\hat{\theta}^{0}$ using for instance the LSADE estimator and use this to define the initial prefilter $\hat{F}\left(p_{x}, p_{t}, \hat{\theta}^{0}\right)=\frac{1}{A\left(p_{x}, p_{t}, \hat{\theta}_{\mathrm{LSADE}}\right)}$, iter $=1$.

\section{- Step 2}

Compute an estimate of the noise-free output $\hat{y}(x, t)$ by simulating the auxiliary model

$$
\hat{A}\left(p_{x}, p_{t}, \hat{\theta}^{i t e r-1}\right) \hat{\grave{y}}(x, t)=0
$$

subjected to initial and boundary conditions and based on the estimated parameters $\hat{\theta}^{i t e r-1}$

\section{- Step 3}

Compute

$$
\begin{gathered}
\left\{\begin{array}{l}
y_{\mathrm{f}}^{\left(i_{x}, i_{t}\right)}(x, t)=F^{\left(i_{x}, i_{t}\right)}\left(p_{x}, p_{t}, \hat{\theta}^{i t e r-1}\right) y(x, t) \\
\hat{\hat{y}}_{\mathrm{f}}^{\left(i_{x}, i_{t}\right)}(x, t)=F^{\left(i_{x}, i_{t}\right)}\left(p_{x}, p_{t}, \hat{\theta}^{i t e r-1}\right) \hat{y}(x, t)
\end{array}\right. \\
F^{\left(i_{x}, i_{t}\right)}\left(p_{x}, p_{t}, \hat{\theta}^{i t e r-1}\right)=\frac{p^{i_{x}} p^{i_{t}}}{\hat{A}\left(p_{x}, p_{t}, \hat{\theta}^{i t e r-1}\right)}
\end{gathered}
$$

with $i_{x}=0,1,2$ and $i_{t}=0,1$.

Prefilter the output $y\left(x_{\ell}, t_{k}\right)$ and auxiliary model output $\hat{\grave{y}}\left(x_{\ell}, t_{k}\right)$ by the filter $F^{\left(i_{x}, i_{t}\right)}$

\section{- Step 4}

Form the filtered estimated regressor as

$$
\varphi_{\mathrm{f}}^{T}\left(x_{\ell}, t_{k}\right)=\left[y_{\mathrm{f}}^{(2,0)}\left(x_{\ell}, t_{k}\right)-y_{\mathrm{f}}^{(1,0)}\left(x_{\ell}, t_{k}\right)\right]
$$

along with the instrument

$$
\zeta_{\mathrm{f}}^{T}\left(x_{\ell}, t_{k}\right)=\left[\hat{\hat{y}}_{\mathrm{f}}^{(2,0)}\left(x_{\ell}, t_{k}\right)-\hat{\grave{y}}_{\mathrm{f}}^{(1,0)}\left(x_{\ell}, t_{k}\right)\right]
$$

Compute the IV estimate

$$
\begin{aligned}
\hat{\theta}_{\text {SRIVADE }}^{i t e r}= & {\left[\sum_{\ell=0}^{L} \sum_{k=0}^{N} \zeta_{\mathrm{f}}\left(x_{\ell}, t_{k}\right) \varphi_{\mathrm{f}}^{T}\left(x_{\ell}, t_{k}\right)\right]^{-1} \cdot } \\
& {\left[\sum_{\ell=0}^{L} \sum_{k=0}^{N} \zeta_{\mathrm{f}}\left(x_{\ell}, t_{k}\right) y_{\mathrm{f}}^{(0,1)}\left(x_{\ell}, t_{k}\right)\right] }
\end{aligned}
$$

where $\hat{\theta}_{\text {SRIVADE }}^{i t e r}$ is the SRIVADE estimate at iteration iter based on filtered data. If convergence occurs or the maximum number of iterations is reached then stop, else set iter $=$ iter +1 and go to Step 2 .

\section{NUMERICAL IMPLEMENTATION OF THE FILTERING OPERATIONS}

Filtering and model simulation involved in the LSADE and SRIVADE methods imply the resolution of PDE. However, the exact solution of a PDE is a continuous function which cannot be directly computed by an exact solution. One solution is to estimate an approximate function using digital methods and considering the non-uniformity of the signals. In the literature, three main methods allow to transform the continuous problem into a discrete one. The first method called finite difference (see e.g. Morton and Mayers [2005]) consists to replace partial derivatives by combination of unknown function values in certain points close to some of the others. This method is simple to establish with moderate computation costs, but the study domain has to be simple to mesh. A second method, the finite volume (see e.g. Leveque [2002]) method consists to directly integrate the PDE on the mesh. This method is interesting when the mesh is not structured, however the convergence is not easy to prove. A third method, the finite element method (see e.g. Claes [1987]) is obtained by the weak formulation of the equation. It allows to take into account several kinds of mesh and to keep the algorithm convergent. Nevertheless this method is quite complicated to implement and implies a large computational load.

In this paper, the finite difference method is chosen to handle resolution of PDE. This technique is based on the use of Taylor polynomial of a function to compute its partial derivative approximation at the point $\left(x_{\ell}, t_{k}\right)$.

\subsection{Approximation from Taylor expansion of degree 2}

The second degree Taylor polynomial approximation of a function $f$ at $x=x_{\ell}$ is defined as

$$
\left\{\begin{array}{r}
f\left(x_{\ell}+h_{\ell+1}\right)=f\left(x_{\ell}\right)+h_{\ell+1} f^{\prime}\left(x_{\ell}\right) \\
+\frac{h_{\ell+1}^{2}}{2} f^{\prime \prime}\left(x_{\ell}\right)+\mathrm{O}\left(h_{\ell+1}^{2}\right) \\
f\left(x_{\ell}-h_{\ell}\right)=f\left(x_{\ell}\right)-h_{\ell} f^{\prime}\left(x_{\ell}\right)+\frac{h_{\ell}^{2}}{2} f^{\prime \prime}\left(x_{\ell}\right)+\mathrm{O}\left(h_{\ell}^{2}\right)
\end{array}\right.
$$

where $\mathrm{O}\left(h_{\ell+1}^{2}\right)$ and $\mathrm{O}\left(h_{\ell}^{2}\right)$ are the local truncation errors which are proportional to the step size.

By algebraic manipulation, some Euler's approximation schemes can be used to approach the first order derivative. An example is given by the backward difference:

$$
f^{\prime}\left(x_{\ell}\right) \approx \frac{f\left(x_{\ell}\right)-f\left(x_{\ell-1}\right)}{h_{\ell}}
$$

where $x_{\ell-1}=x_{\ell}-h_{\ell}$.

Following the same idea, the second derivative can be approached by the central difference:

$$
\begin{aligned}
f^{\prime \prime}\left(x_{\ell}\right) \approx \frac{f\left(x_{\ell+1}\right)-f\left(x_{\ell}\right)}{h_{\ell+1}^{2}}+ & \frac{f\left(x_{\ell-1}\right)-f\left(x_{\ell}\right)}{h_{\ell}^{2}} \\
& +\left(\frac{1}{h_{\ell}}-\frac{1}{h_{\ell+1}}\right) f^{\prime}\left(x_{\ell}\right)
\end{aligned}
$$

where $x_{\ell+1}=x_{\ell}+h_{\ell+1}$.

\subsection{Simulation of the ADE model}

To illustrate the method described above, let us consider (24) written in developed form

$$
\left\{\begin{array}{l}
\frac{\partial \stackrel{\circ}{y}(x, t)}{\partial t}=\hat{a}_{20} \frac{\partial^{2} \stackrel{\circ}{y}(x, t)}{\partial x^{2}}-\hat{a}_{10} \frac{\partial \stackrel{\circ}{y}(x, t)}{\partial x} \\
\stackrel{\text { on } \left.] 0, x_{\ell}[\times] 0, T\right]}{y(x, t)}=\stackrel{\circ}{u}_{\Gamma}(x, t) \text { on }\left\{0, x_{\ell}\right\} \times[0, T] \\
\left.\stackrel{\circ}{y}(x, 0)=\stackrel{y}{y}_{0}(x) \text { on }\right] 0, x_{\ell}[
\end{array}\right.
$$

with the following boundary conditions

$$
\stackrel{\circ}{u}_{\Gamma}(x, t)=\left\{\begin{array}{l}
\stackrel{\circ}{u}_{\Gamma_{1}}(t)=u(t) \forall t \in[0, T] \text { if } x=0 \\
\stackrel{\circ}{u}_{\Gamma_{\mathrm{r}}}(t)=0 \forall t \in[0, T] \text { if } x=x_{L}
\end{array}\right.
$$


and the initial condition

$$
\left.\stackrel{\circ}{y}_{0}(x)=0 \forall x \in\right] 0, x_{\ell}[
$$

Using a forward difference at time $t_{k}$ and the derivative approximations (31) and (32), a recurrence equation is used to approximate the PDE in (33) as:

$$
\begin{aligned}
& \frac{\stackrel{\circ}{\ell}_{\ell}^{k+1}-\stackrel{\circ}{y}_{\ell}^{k}}{T_{s}}=\hat{a}_{20}\left[\frac{\stackrel{\circ}{y}_{\ell+1}^{k}-\stackrel{\circ}{y}_{\ell}^{k}}{h_{\ell+1}^{2}}+\frac{\stackrel{\circ}{y}_{\ell-1}^{k}-\stackrel{\circ}{y}_{\ell}^{k}}{h_{\ell}^{2}}\right. \\
& \left.+\left(\frac{1}{h_{\ell}}-\frac{1}{h_{\ell+1}}\right)\left(\frac{\stackrel{\circ}{y}_{\ell}^{k}-\grave{ }_{\ell-1}^{k}}{h_{\ell}}\right)\right]-\hat{a}_{10} \frac{\stackrel{\circ}{y}_{\ell}^{k}-\stackrel{\circ}{y}_{\ell-1}^{k}}{h_{\ell}}
\end{aligned}
$$

with $\ell=\{1, \cdots, L-1\}, k=\{0, \cdots, N-1\}$. The points $\dot{y}_{\ell}^{k}$ represent the numerical approximations of $\stackrel{y}{y}\left(x_{\ell}, t_{k}\right)$.

The boundary and initial conditions are discretized by:

$$
\left\{\begin{array}{l}
{\stackrel{\circ}{\Gamma}}_{\Gamma_{1}}\left(t_{k}\right)=u^{k} \forall k \in\{0, \cdots, N\} \\
\stackrel{\circ}{u}_{\Gamma_{\mathrm{r}}}\left(t_{k}\right)=0 \forall k \in\{0, \cdots, N\} \\
\stackrel{\circ}{y}_{0}\left(x_{\ell}\right)=0 \forall \ell \in\{1, \cdots, L-1\}
\end{array}\right.
$$

The scheme (36) can be rewritten as follow:

$$
\begin{gathered}
\dot{\circ}_{\ell}^{k+1}=\alpha_{-1}\left(h_{\ell}, h_{\ell-1}\right) \grave{y}_{\ell-1}^{k}+\alpha_{0}\left(h_{\ell}, h_{\ell-1}\right) \dot{y}_{\ell}^{k} \\
+\alpha_{1}\left(h_{\ell}, h_{\ell-1}\right) \dot{y}_{\ell+1}^{k}
\end{gathered}
$$

where $\alpha_{-1}, \alpha_{0}$ and $\alpha_{1}$ are varying-coefficients depending on the spacial sampling:

$$
\begin{aligned}
& \alpha_{-1}=\hat{a}_{20} T_{s}\left[\frac{1}{h_{\ell}^{2}}-\left(\frac{1}{h_{\ell}}-\frac{1}{h_{\ell+1}}\right) \frac{1}{h_{\ell}}\right]+\frac{\hat{a}_{10} T_{s}}{h_{\ell}} \\
& \alpha_{0}=1-\hat{a}_{20} T_{s}\left[\left(\frac{1}{h_{\ell}^{2}}+\frac{1}{h_{\ell+1}}\right)-\left(\frac{1}{h_{\ell}}-\frac{1}{h_{\ell+1}}\right) \frac{1}{h_{\ell}}\right] \\
& -\frac{\hat{a}_{10} T_{s}}{h_{\ell}} \\
& \alpha_{1}=\frac{\hat{a}_{20} T_{s}}{h_{\ell+1}^{2}}
\end{aligned}
$$

\section{Comments}

- The digital implementation of the various continuous filtering operations (see equations 25) is achieved using the same discretization methods.

- From the discrete formulation (38), equation (33) can be identified using a refined IV approach with parameter-varying model (see Ali et al. [2011]). However missing data reconstruction by linear interpolation of the parameters is not always possible in this case of non-uniformly sampling. The proposed approach in this paper is adapted to the missing point estimation, and presents moreover the advantage of requiring less parameters to be estimated.

\section{NUMERICAL EXAMPLES}

In this section, the ADE is used as example with the same conditions illustrated in Section 4.2. The excitation signal $\stackrel{\circ}{u}_{\Gamma_{1}}$ is not distributed spatially, and is considered as a boundary condition (see Fig. 2). It represents the upstream evolution of a solute concentration in time.

The signal $\stackrel{\circ}{y}\left(x_{\ell}, t_{k}\right)$ represents the solute propagation in the river. This signal is corrupted by a two dimensional,

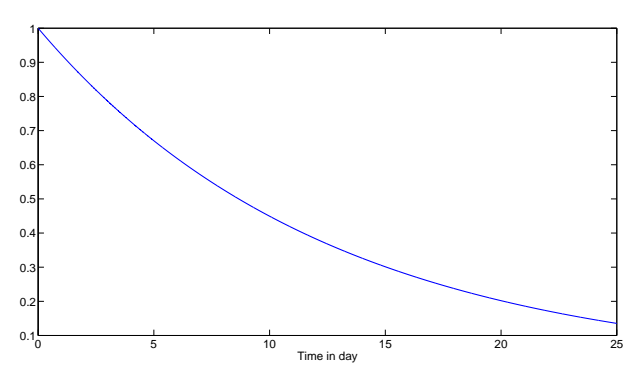

Fig. 2. Boundary conditions for $x=0$

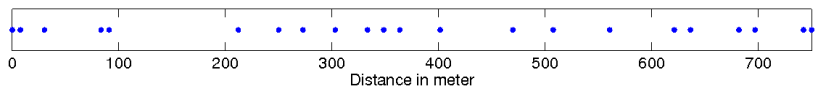

Fig. 3. Sensors distribution (blue point) (case 2)

zero-mean and normally distributed, discrete-time noise signal, with a Signal-to-Noise Ratio (SNR) defined as

$$
S N R=10 \log _{10}\left(\frac{P_{e}}{P_{\grave{y}}}\right)
$$

where $P_{e}$ and $P_{y}$ are the average power of the noise and deterministic output. Two Monte Carlo simulation analysis are used to illustrate and compare the performance of two approaches to ADE model identification discussed in the paper.

- The first simulation considers the signal $\stackrel{\circ}{y}\left(x_{\ell}, t_{k}\right)$ with a uniform sampling. This case represents the ideal case where all spatial locations and time-instants are available to identify the model parameters. The time sampling is $T_{s} \approx 72 \mathrm{~s}$ and $N=30000$ and the space sampling is $h_{\ell} \approx 7.5 \mathrm{~m}$ with $L=100$.

- The second simulation investigates the case where the signal is measured at a few non-uniformly distributed spatial points only. Here, the time sampling is constant as in Case 1 but only 20 spatial locations, randomly chosen amongst the 100 available space points, are used to estimate the model parameters. The sensors are distributed non-uniformly as showed in Fig. 3. This results in non-uniformly, fairly coarsely sampled spatial data, which is more representative of practical situations where spatial measurements, in particular, can be difficult.

\subsection{Case 1: Uniformly sampled data situation}

In Fig. 4, the noise-free data are showed for all the space points.

Table 1. $\mathrm{MC}$ simulation results, $\mathrm{SNR}=35 \mathrm{~dB}$ (case 1)

\begin{tabular}{|c|c|c|c|}
\hline \multicolumn{2}{|c|}{} & $\hat{a}_{20}$ & $\hat{a}_{10}$ \\
\hline Method & True value & 0.26 & -0.001917 \\
\hline \hline \multirow{2}{*}{ LSADE } & mean & 0.092027 & -0.002429 \\
\cline { 2 - 4 } & std & 0.011916 & $3.6647 \cdot 10^{-5}$ \\
\hline \hline \multirow{2}{*}{ SRIVADE } & mean & 0.26001 & -0.001917 \\
\cline { 2 - 4 } & std & $8.8887 \cdot 10^{-5}$ & $7.3084 \cdot 10^{-7}$ \\
\hline
\end{tabular}

The unknown parameters $\hat{a}_{20}$ and $\hat{a}_{10}$ are estimated by the least squares based SVF (denoted as LSADE) and the 


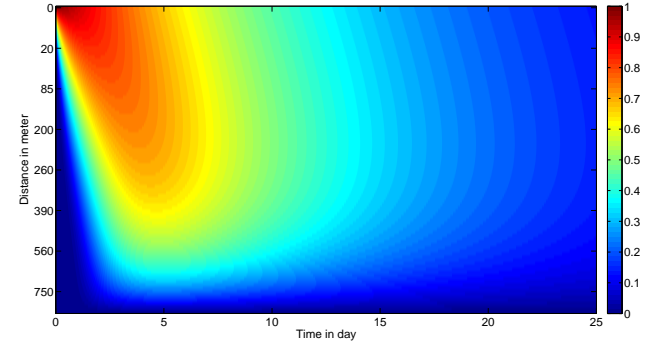

Fig. 4. Noise-free data - Uniformly sampling (case 1)

proposed SRIVADE methods.

From Table 1, it clearly appears that the LSADE method provides, as expected, biased parameter estimates. On the contrary, the SRIVAPE method gives unbiased estimates with low variance. It clearly outperforms the LS method in the case where the noise is supposed to be white. These results depict the ideal case where the spatial data number is not limited.

\subsection{Case 2: Non-uniformly spatial sampling situation}

Only 20 non-uniformly distributed space points are used in this second example and the resulting noise-free data are illustrated in Fig. 5

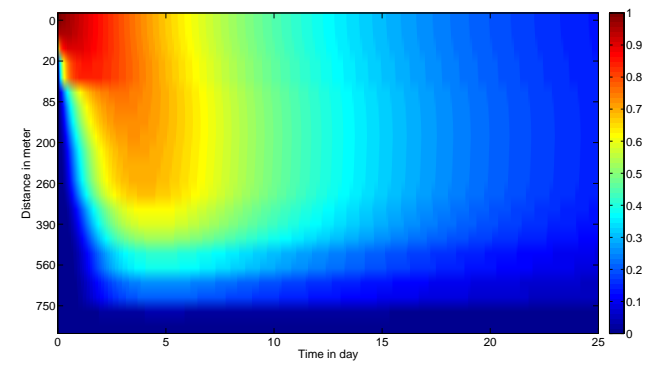

Fig. 5. Noise-free data - Non-uniformly sampling (case 2)

Table 2. $\mathrm{MC}$ simulation results, $\mathrm{SNR}=25 \mathrm{~dB}$ (case 2)

\begin{tabular}{|c|c|c|c|}
\hline \multicolumn{2}{|c|}{} & $\hat{a}_{20}$ & $\hat{a}_{10}$ \\
\hline Method & True value & 0.26 & -0.001917 \\
\hline \hline \multirow{2}{*}{ LSADE } & mean & 0.16891 & -0.002151 \\
\cline { 2 - 4 } & std & 0.0423 & $1.2486 \cdot 10^{-4}$ \\
\hline \hline \multirow{2}{*}{ SRIVADE } & mean & 0.2601 & -0.001918 \\
\cline { 2 - 4 } & std & $8.9963 \cdot 10^{-4}$ & $7.6603 \cdot 10^{-6}$ \\
\hline
\end{tabular}

The Monte Carlo simulation results displayed in Table 2 show that, in this case where the spatial sampling is coarser and non-uniform, the resulting LSADE estimates are once again biased while the SRIVADE method still delivers unbiased estimates with very small standard deviations. It has to be noticed that this case represents the most practical experiment. Indeed, a large number of temporal data is an easy matter considering nowadays acquisition capabilities. Nevertheless, increasing spatial data is equivalent to increasing the number of sensors, consequently the number of available data should remain in practical physical range.

\section{CONCLUSION}

This paper has presented an estimation method for identifying the advection-diffusion equation when the data are non uniformly distributed in space. The proposed technique is based on a refined instrumental variable algorithm. This method involves an Euler's method to approach the solution of the advection-diffusion equation when the space points are not uniformly distributed. Because it is likely in practical situations, such as those encountered in environmental research and development, that the spatial sampling strategy will be constrained, the sensitivity of the estimation scheme to coarser, nonuniform spatial sampling has been analyzed in this paper. A Monte Carlo simulation based on the practical case of the diffusion of a solute concentration along a river has been used to illustrate the effectiveness of the proposed solution.

\section{REFERENCES}

M. Ali, A. Ali, H. Abbas, and H. Werner. Identification of BoxJenkins models for parameter-varying spatially interconnected systems. American Control Conference (ACC), San Francisco (USA), pages 145-150, 2011.

J. Claes. Numerical Solution of Partial Differential Equations by the Finite Element Method. Cambridge University Press, 1987.

D. Coca and S.A. Billings. Identification of coupled map lattice models of complex spatio-temporal patterns. Physics Letters A, 287:65-73, 2001.

Z. Hidayat, A. Núñez, R. Babušk, and B. De Schutter. Identification of distributed-parameter systems with missing data. IEEE International Conference on Control Applications, Part of 2012 IEEE Multi-Conference on Systems and Control, pages 10141019, Dubrovnik, Croatia 2012.

R. J. Leveque. Finite volume methods for hyperbolic problems. Cambridge University Press, 2002.

C. J. Martinez and W. R. Wise. Analysis of constructed treatment wetland hydraulics with the transient storage model otis. Ecological Engineering, 20:211-222, 2003.

K. W. Morton and D. F. Mayers. Numerical Solution of Partial Differential Equations. Cambridge University Press, 2005.

T. K. Nilssen, K. H. Karlsen, T. Mannseth, and X.-C. Tai. Identification of diffusion parameters in a nonlinear convection-diffusion equation using the augmented lagrangian method. Computational Geosciences, 13(3):317-329, April 2009.

J. Schorsch, H. Garnier, and M. Gilson. Instrumental variable methods for identifying partial differential equation models of distributed parameter systems. In IFAC Symposium on system identification, pages 840-845, Brussels (Belgium), 2012.

T. Söderström and P. Stoica. Instrumental Variable Methods for System Identification. Springer Verlag, New York, 1983.

W. Tych and P.C. Young. A matlab software framework for dynamic model emulation. Environmental Modelling and Software, 34:1929, 2012.

P.C. Young. Recursive estimation and time-series analysis. Springer-Verlag, Berlin, 1984.

P.C. Young. Recursive Estimation and Time-series Analysis: An Introduction for the Student and Practitioner. Springer-Verlag, Berlin, second edition, 2011.

P.C. Young and A.J. Jakeman. Refined instrumental variable methods of time-series analysis: Part III, extensions. International Journal of Control, 31:741-764, 1980.

P.C. Young, H. Garnier, and M. Gilson. Identification of continuoustime models from sampled data, chapter Refined instrumental variable identification of continuous-time hybrid Box-Jenkins models, pages 91-132. Springer-Verlag, London, H. Garnier and L. Wang (Eds.) edition, 2008. 